\title{
Prevalência de parasitas intestinais em escolares provenientes de dois municípios da Bahia, Brasil
}

As parasitoses intestinais causadas por helmintos e protozoários acometem várias pessoas, as mais infectadas são as crianças em idade escolar contribuindo para o agravamento dos problemas de saúde pública, principalmente nos países subdesenvolvidos e em desenvolvimento. O objetivo deste trabalho foi diagnosticar parasitas intestinais provenientes de crianças de 05 a 15 anos de idade provenientes das cidades de Salvador e Paulo Afonso, Bahia - Brasil. O estudo foi realizado no período de fevereiro de 2013 a dezembro de 2014 . Foram coletadas 139 amostras fecais de escolares oriundos de Salvador e 106 amostras fecais de Paulo Afonso totalizando 245 amostras. O método de sedimentação espontânea foi utilizado para os exames das amostras fecais. Os resultados dos exames revelaram que dentre os parasitas intestinais os mais frequentes nas amostras das crianças foram os protozoários Endolimax nana com $19,38 \%$ seguido de Entamoeba coli com 10,2\% e Blastocystis spp. que apresentou $8,6 \%$ do total das amostras coletadas e analisadas. No município de Paulo Afonso os protozoários Endolimax nana seguido de Entamoeba coli com 10,2\% e Blastocystis spp. que apresentou 8,6\% do total das amostras coletadas e analisadas. No município de Paulo Afonso os protozoários Endolimax nana
$(12,69 \%)$, Blastocystis spp. e Giardia lamblia ambas com $(10,31 \%)$ e Entamoeba coli $(7,14 \%)$ foram os mais prevalentes. Os helmintos diagnosticados no município de Salvador foram Ascaris (12,69\%), Blastocystis spp. e Giardia lamblia ambas com $(10,31 \%)$ e Entamoeba coli $(7,14 \%)$ foram os mais prevalentes. Os helmintos diagnosticados no município de Salvador foram Ascaris
lumbricoides $(9,13 \%)$, Trichuris trichiura $(3,76 \%)$ e Ancilostomídeo $(2,15 \%)$, já no município de Paulo Afonso os achados foram Ascaris lumbricoides $(6,3 \%)$, Ancilostomídeo e Hymenolepis nana com (1,58\%) e Trichuris trichiura (0,79\%). Quanto a múltiplas infecções por parasitos observou-se os seguintes percentuais: monoparasitadas $31,65 \%$ oriundos de Salvador e $31,14 \%$ de Paulo Afonso; poliparasitadas com 23,05\% de Salvador e Paulo Afonso com 16,98\%. Em relação a frequência de enteroparasitoses por faixa etária, no município de Salvador constatou-se mais ocorrente na faixa etária de 9-11 anos com 39,04\% e no município de Paulo Afonso com a faixa etária $5-7$ anos com $42,85 \%$. Em relação a frequência de parasitoses por sexo, o feminino foi o mais infectado com 28,06\% na cidade de Salvador e com 25,47\% em Paulo Afonso e também com o maior percentual de poliparasitismo por sexo com 53,12\% em Salvador em Paulo de pesquisas mais aprofundadas e exames de fezes de rotina com faixa etária que corresponde as crianças de 5 a 13 anos nas cidades pesquisadas, pelo alto índice de infecções neste seguimento bem como indica possíveis problemas socioeconômicos na região estudada.

\section{Prevalence of intestinal parasites in schoolchildren from two municipalities in Bahia, Brazil}

\begin{abstract}
Intestinal parasitic infections caused by helminths and protozoa affect several people, the most infected being school-age children contributing to the worsening of public health problems, especially in underdeveloped and developing countries. The aim of this study was to diagnose intestinal parasites from children aged 5 to 15 years old from the cities of Salvador and Paulo Afonso, Bahia - Brazil. The study was carried out from February 2013 to December 2014. 139 fecal samples were collected from schoolchildren from Salvador and 106 fecal samples from Paulo Afonso totaling 245 samples. The spontaneous sedimentation method was used to examine fecal samples. The results of the tests revealed that among the intestinal parasites the most frequent in the children's samples were the protozoa Endolimax nana with $19.38 \%$, followed by Entamoeba coli with $10.2 \%$ and Blastocystis spp. which presented $8.6 \%$ of the total samples collected and analyzed. In the city of Paulo Afonso the protozoa Endolimax nana (12.69\%), Blastocystis spp. and Giardia lamblia both with (10.31\%) and Entamoeba coli (7.14\%) were the most prevalent. The helminths diagnosed in the municipality of Salvador were Ascaris lumbricoides (9.13\%), Trichuris trichiura (3.76\%) and Hookworms (2.15\%), while in Paulo Afonso the findings were Ascaris lumbricoides (6.3\%) Hookworm and Hymenolepis nana com (1.58\%) and Trichuris trichiura (0.79\%). As for multiple parasite infections, the following percentages were hinins observed: $31.65 \%$ m enteroparasitosis by age group, in the municipality of Salvador it was found more frequent in the age group of 9-11 years old with $39.04 \%$ and in the city of Paulo Afonso with the age group 5-7 years old with $42.85 \%$. Regarding the frequency of parasites by sex, the female was the most infected with $28.06 \%$ in the city of Salvador and with $25.47 \%$ in Paulo Afonso and also with the highest percentage of polyparasitism by sex with $53.12 \%$ in Salvador in Paulo Afonso female and male both with $50 \%$. It is concluded that in the studied samples a considerable portion of infected children were found. And these data suggest the conduct of more in-depth research and routine stool examinations with an age group that corresponds to children aged 5 to 13 years in the cities surveyed, due to the high rate of infections in this segment as well as indicating possible socioeconomic problems in the studied region.
\end{abstract}

Keywords: Intestinal parasites; Children; School; Poliparasitism.

Topic: Parasitologia

Reviewed anonymously in the process of blind peer.
Received: 05/06/2020

Approved: 10/08/2020
Marilene Aparecida Moreira (iD)

Universidade do Estado de Mato Grosso, Brasil http://lattes.cnpq.br/1901063422457798

http://orcid.org/0000-0002-1943-9000

imhemala@hotmail.com

Yasmin Fernanda Nunes Viana (iD)

Universidade do Estado de Mato Grosso, Brasil

http://lattes.cnpq.br/7288980470044764

http://orcid.org/0000-0001-8740-7089

yasminfnv@hotmail.com

\section{Gilliard Lima (iD)}

Universidade do Estado de Mato Grosso, Brasil http://lattes.cnpq.br/8272144638438444 http://orcid.org/0000-0003-3914-0572 gilliard s.lima@hotmail.com
Tatiane Amorim de Matos (iD)

Universidade do Estado de Mato Grosso, Brasil http://lattes.cnpq.br/5844726893235718 http://orcid.org/0000-0002-2898-1897 tianeamatos@gmail.com

Bianca Teshima de Alencar (iD

Universidade do Estado de Mato Grosso, Brasil http://lattes.cnpq.br/9101535074774508 http://orcid.org/0000-0001-6812-3494 biateshima@hotmail.com

\section{Aireno de Souza Silva (id}

Universidade do Estado de Mato Grosso, Brasil http://lattes.cnpq.br/3376672192214087 http://orcid.org/0000-0002-4404-705X aireno cac@hotmail.com

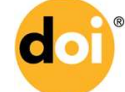

DOI: $10.6008 / C B P C 2236-9600.2020 .003 .0002$
Referencing this:

MOREIRA, M. A.; VIANA, Y. F. N.; LIMA, L. G.; MATOS, T. A.; ALENCAR, B. T.; SILVA, A. S.; MALHEIROS, A. F.. Prevalência de parasitas intestinais em escolares provenientes de dois municípios da Bahia, Brasil. Scire Salutis, v.10, n.3, p.12-20, 2020. DOI:

http://doi.org/10.6008/CBPC2236-9600.2020.003.0002 


\section{INTRODUÇÃO}

Parasitoses intestinais são consideradas um problema de saúde pública, especialmente em países subdesenvolvidos e em desenvolvimento, sendo a doença mais comum no mundo com uma prevalência de 25\% na população humana. Estando a sua transmissão dependente das condições sanitárias e de higiene dos indivíduos (SBMFC, 2019).

As parasitoses intestinais são infecções ou infestações que possuem como causa helmintos e protozoários que habitam o trato intestinal, sendo uma patologia comum no mundo e endêmica em países de terceiro mundo e em certas regiões do Brasil. Quando o indivíduo apresenta positividade para 3 ou mais parasitas (poliparasitismo) influência diretamente nas taxas de morbidade e mortalidade (FIOCRUZ, 2019).

Segundo dados do Departamento de Informática do Sistema Único de Saúde (DATASUS), no ano de 2014 as patologias infecciosas e parasitárias representaram a sexta causa de morbidade no país, o que se traduz em 776.358 internações, representando 7,28\% da morbidade hospitalar no período (SANTOS et al., 2017). A infecção parasitaria apresenta uma ampla distribuição geográfica, afetando áreas urbanas e rurais, com variações de acordo com o ambiente e espécie de parasita envolvido (SANTOS et al., 2017).

Os helmintos e protozoários que mais aparecem entre as parasitoses intestinais podem ser transmitidos principalmente pela água contaminada ou solo. Dos helmintos fazem parte as patologias; ascaridíase, enterobíase, estrongiloidíase, ancilostomíase, tricuríase, teníase, himenolepíase e esquistossomose. Já dos protozoários fazem parte as patologias; giardíase, amebíase, balantidíase e criptosporidíase (FIOCRUZ, 2019).

Os sintomas das parasitoses intestinais podem variar de acordo com as condições de vida, moradia e saneamento básico do paciente podendo chegar a casos leves como anorexia, irritabilidade, distúrbio do sono, náuseas, vômitos e diarreia, podendo também apresentar sintomas mais graves como disenteria, prurido anal, desnutrição, presença de sangue nas fezes, anemia ferropriva, eliminação e vermes cilíndricos nas fezes ou vômitos e obstrução intestinal (FIOCRUZ, 2019).

Quando acometem crianças acarretam inúmeros prejuízos para a saúde das mesmas, uma vez que não possuem uma defesa imunológica eficaz contra esses parasitas. Dentre essas alterações estão o comprometimento físico, mental e social, dificultando seu desenvolvimento e crescimento (NAVONE et al., 2017). O impacto negativo da infecção por parasitoses produz, além da redução no desenvolvimento físico e mental, uma diversidade de quadros mórbidos que incluem diarreia, dores abdominais, perda de peso, até complicações como a formação de granulomas e processos obstrutivos que exigem intervenção cirúrgica, podendo inclusive levar o paciente ao óbito (BRASIL, 2016).

O clima quente e úmido, a pobreza, a desnutrição, a falta de água limpa, a diversidade e a densidade da população, os baixos níveis de higiene, o contato próximo com os reservatórios infectados e a falta de pesquisa contínua são consideradas como fatores importantes na ocorrência e disseminação das doenças causadas por parasitas intestinais (SHAHDOUST et al., 2016).

Considerando a grave repercussão na saúde de crianças acometidas por parasitas intestinais e que 
as presenças de enfermidades parasitárias servem como importantes indicadores problemas socioeconômicos de um local, o presente estudo teve como objetivo estabelecer a ocorrência de parasitas intestinais em escolares entre 5 a 15 anos oriundos dos municípios de Salvador e Paulo Afonso no estado da Bahia.

\section{MATERIAIS E MÉTODOS}

Trata-se de um estudo epidemiológico de corte transversal. As regiões de coletas das amostras fecais ocorreram nas cidades de Salvador e Paulo Afonso, municípios do estado da Bahia no período de fevereiro de 2013 a dezembro de 2014. Economicamente Salvador é uma das mais relevantes do país. Desde a sua fundação, Salvador tem sido um dos portos e centros de comércio internacional mais importante do Brasil. Com uma grande refinaria de petróleo, uma planta petroquímica e outras indústrias importantes em seu território, a cidade tem feito grandes progressos na redução da sua dependência histórica da agricultura para a sua prosperidade econômica. Possui um clima tropical predominantemente quente, com chuvas no inverno e verão seco, chega a extremos de $15^{\circ} \mathrm{C}$ no inverno e a $38^{\circ} \mathrm{C}$ no verão. A brisa oriunda do Oceano Atlântico deixa agradável a temperatura da cidade mesmo nos dias mais quentes (IBGE, 2010).

A cidade de Paulo Afonso pertence à microrregião homogênea no 147 - Sertão de Paulo Afonso - e ocupa uma área territorial de 1.579,722 quilômetros quadrados. De acordo com o censo do IBGE (2010) tem uma população de 108.396 mil pessoas. Por estar situado na área do Sertão Nordestino, Paulo Afonso possui clima tipo semiárido, com pluviosidade média entre 500 e 600 mililitros anuais. A temperatura média elevada

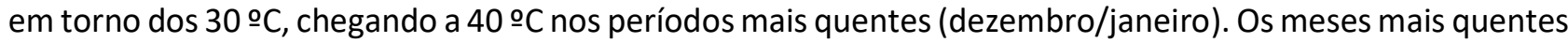
são de outubro a janeiro e julho é o mais frio com temperatura em torno de 22 ㄷ (IBGE, 2010).

A população alvo da pesquisa foram crianças de 5 a 15 anos (idade escolar), selecionadas aleatoriamente que desejassem participar da pesquisa e entregassem devidamente as amostras fecais. A análise das amostras fecais ocorreu em quatro etapas: $1^{\circ}$ conservação em etanol $70 \%$ e a $2^{\circ}$ em Dicromato de Potássio $2 \%$ que foram devidamente armazenadas sob temperatura a $4^{\circ} \mathrm{C}$. A $3^{\circ}$ etapa onde ocorreu a alíquota de cada amostra que foi posteriormente congelada a -20 ㄷ C para conservação dos parasitas intestinais e a $4^{\circ}$ etapa na qual as amostras foram analisadas por meio da técnica de sedimentação espontânea de Hoffmann. Sendo utilizada a solução Lugol para a coloração das lâminas.

Os resultados dos dados foram expressos em números absolutos e frequência, apresentados em forma de tabelas e gráficos. A presente pesquisa integra o projeto maior "Biodiversidade das parasitoses intestinais provenientes de amostras fecais humanas e de cães dos seis biomas brasileiros: caracterização parasitológica e molecular", desenvolvido pela Universidade do Estado de Mato- Grosso em parceria com o Ministério da Saúde, e sob aprovação do comitê de Ética em Pesquisa da Universidade de São Paulo (CEP/USP), com o parecer: 310.501-CEP/USP.

\section{RESULTADOS E DISCUSSÃO}

Foram analisadas um total de 245 amostras fecais, com o percentual de $51,84 \%$ de casos positivos 
para os enteroparasitas e $48,16 \%$ de casos negativos. Na cidade de Salvador observou-se um percentual de 54,67\% de casos positivos para 45,33\% de casos negativos e na cidade de Paulo Afonso com 48,12\% de positividade e $51,88 \%$ de negatividade (tabela 1 ).

Constata se que este percentual é alto em relação a outros estudos de variadas regiões do país. AULER et al. (2018), encontrou positividade de 13,9\% $(n=40)$ para parasitas intestinais em crianças de 2 a 5 anos no município de Guarapuava/PR. Segundo Antunes et al. (2017), foram encontrados no total de $18 \%$ de casos positivos no município de Santo Ângelo/RS na cidade de Campo Andrade et al. (2017) encontraram o índice de positividade de $25 \%$ no total. As discordâncias com o atual estudo podem ser explicadas pelas diferenças socioeconômicas nos grupos pesquisados, bem como o modo de vida diária de cada região.

Tabela 1: Prevalência de parasitas intestinais em crianças oriundos dos municípios de Salvador e Paulo Afonso, BA.

\begin{tabular}{lllllll}
\hline \multirow{2}{*}{ Cidades } & \multicolumn{2}{l}{ Casos Positivos } & \multicolumn{2}{l}{ Casos Negativos } & \multicolumn{2}{l}{ Total } \\
\cline { 2 - 8 } & $\boldsymbol{N}$ & $\boldsymbol{\%}$ & $\boldsymbol{n}$ & $\boldsymbol{\%}$ & $\boldsymbol{N}$ & $\boldsymbol{\%}$ \\
\hline Salvador & 76 & 54,67 & 63 & 45,33 & 139 & 100 \\
\hline Paulo Afonso & 51 & 48,12 & 55 & 51,88 & 106 & 100 \\
\hline Total & 127 & 51,84 & 118 & 48,16 & 245 & 100 \\
\hline
\end{tabular}

Dos casos positivos, $28,06 \%$ das amostras são do sexo feminino e $26,61 \%$ do sexo masculino da cidade de Salvador. Na cidade de Paulo Afonso também foram encontradas alta positividade no sexo feminino com $25,47 \%$ e masculino com $22,5 \%$ (Figura 1). Andrade et al. (2017) estudo realizado CMEI de Campo Mourão/Paraná com 32 crianças, corrobora com relação ao gênero das crianças, observando maior positividade para o gênero feminino com $75 \%(n=6)$.

Segundo Pereira et al. (2018) constatou-se que 38 (40\%) apresentaram positividade para cisto de protozoários e/ou ovos de helmintos. Da amostragem contaminada, ao se comparar a infecção em relação ao sexo, obteve-se que a maior prevalência de parasitoses ocorreu em crianças do sexo masculino, totalizando $19(63,2 \%)$, enquanto que nas crianças do sexo feminino a ocorrência foi 14 (36,8\%). Nossos achados não corroboram com os resultados de Lacerda et al. (2017) que detectou a positividade proporcionalmente ao número de exames realizados por gênero, 6,0 e 9,4\% para os gêneros feminino e masculino respectivamente. Pereira et al. (2018) ao se comparar a contaminação em relação ao sexo, constatou-se que a maior prevalência de infecção parasitária ocorreu em crianças do sexo masculino, totalizando $63,2 \%$, enquanto que nas crianças do sexo feminino a ocorrência foi de $36,8 \%$. Provavelmente, os fatores associados a tal situação diagnosticada sugerem que crianças do sexo masculino interagem mais do que as meninas com o meio ambiente contaminado, andam descalços, não têm hábitos higiênicos, e, culturalmente, as brincadeiras realizadas pelos meninos permitem um maior acesso a sujidade, fato este não muito habitual entre as meninas que costumam brincar mais no interior de suas residências.

Apesar dos estudos citados acima não identificarem a ocorrência maior no sexo feminino e alguns maiores no sexo masculino, deve se levar em conta que as regiões se diferem uma das outras e também fator socioeconômico. Com relação de enteroparasitos encontrados, foi observado maior frequência de protozoários como E. nana 19,38\% , Entamoeba coli 10,2\% e Blastocystis spp. 8,6\% de ambos do município de Salvador. No município de Paulo Afonso encontrou se os protozoários E. nana 12,69\%, Blastocystis spp. e 
G. Iamblia ambas com $10,31 \%$ e E. coli 7,14\%. Os helmintos encontrados no município de Salvador foram A. lumbricoides 9,13\%, T. trichiura 3,76\% e Ancilostomídeo 2,15\%, já no município de Paulo Afonso encontrou se $A$. lumbricoides 6,3\%, Ancilostomídeo e Hymenolepis nana com 1,58\% e T. trichiura 0,79\% conforme verificado na (tabela 2 ).

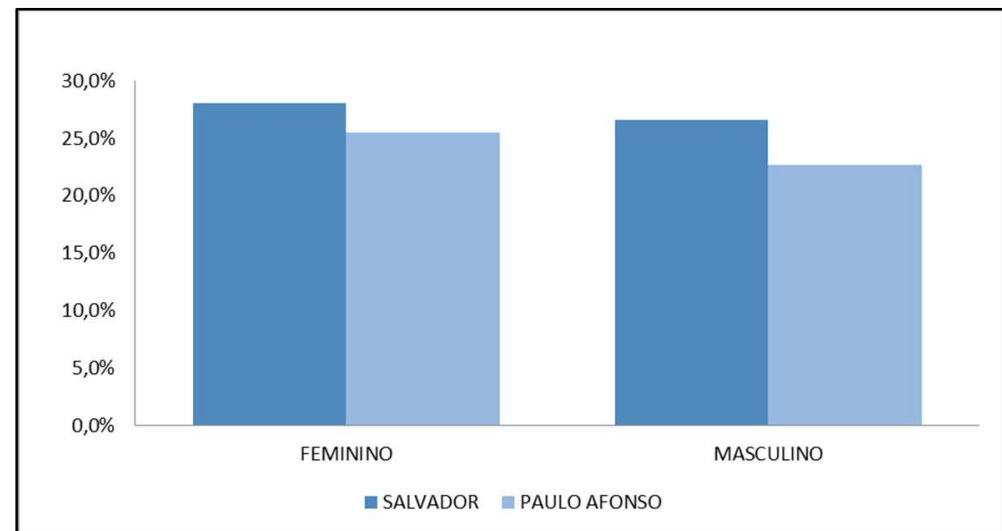

Figura 1: Frequência de parasitas intestinais por sexo provenientes de crianças dos municípios de Salvador e Paulo Afonso, BA.

Pereira et al., (2018) destacaram que a contaminação por ovos de helmintos nas amostras fecais foi similar às quatro espécies detectadas, sendo a contaminação presente apenas em crianças do sexo masculino, onde uma (5,3\%) criança apresentou ovos de Ascaris lumbricoides; uma (5,3\%) com ovos de Hymenolepis nana; uma (5,3\%) apresentou ovos de Ancylostoma sp. e uma (5,3\%) com ovos de Enterobius vermicularis.

Quanto a maior prevalência ter sido por protozoários intestinais em relação aos helmintos, nossos dados corroboram com os estudos realizados por Larceda; Jardim (2017) que identificaram através da análise de 9.520 prontuários de pacientes submetidos à pesquisa de parasitoses por meio de exames coprológicos. Destes, $3 \%(n=312)$ foram positivos para Endolimax nana; $2 \%$ de contaminação $(n=171)$ para Entamoeba coli; e, $3 \%(n=267)$ para a espécie Giardia lamblia. Concomitante também com a pesquisa realizada por Andrade et al. (2017) os exames parasitológicos de fezes das 8 amostras positivas, $75 \%(n=6)$ apresentaram a presença de alguma espécie de protozoários e $25 \%(n=2)$ a presença de helminto. Os protozoários encontrados foram Endolimax nana, Giardia duodenalis e Entamoeba coli, ocorrendo um poliparasitismo em duas amostras com a presença de Giardia duodenalis e Endolimax nana. O único helminto encontrado foi Dipylidium canium.

Em estudo semelhante Auler et al. (2018) revelou que a prevalência das parasitoses mostrou que para o grupo dos protozoários encontramos cistos de $G$. duodenalis $70,4 \%(n=31)$, seguido de Entamoeba coli 11,4\% ( $n=5)$ e Endolimax nana 11,4\% ( $n=5)$ sendo esses últimos considerados protozoários não-patogênicos. Enquanto que, Ascaris lumbricoides 6,8\% $(n=3)$ foi o único helminto encontrado.

Com relação a frequência de enteroparasitoses por faixa etária, no município de Salvador constatou maior ocorrência na faixa etária de 9-11 anos com 39,04\% de casos positivos, $11-13$ anos com 27,80\%, 7-9 anos com 20,33\%, 13-15 anos com 10,69\% e 5-7 anos com 2,14\%, no município de Paulo Afonso a faixa etária com maior ocorrência foi a de 5-7 anos com 42,85\% positivo, 7-9 anos 32,55\%, 9-11 anos com 13, 49\%, 11- 
13 anos 6,35\% e por último 13-15 anos 4,76\% (Figura 2).

Tabela 2: Classificação geral das espécies de enteroparasitos diagnosticados em crianças dos municípios de Salvador e Paulo Afonso, Bahia.

\begin{tabular}{|c|c|c|c|c|}
\hline \multirow[t]{3}{*}{ Parasitas Intestinais } & \multicolumn{2}{|c|}{ Salvador } & \multicolumn{2}{|c|}{ Paulo Afonso } \\
\hline & \multicolumn{4}{|c|}{ Amostra Geral } \\
\hline & $n$ & $\%$ & $n$ & $\%$ \\
\hline \multicolumn{5}{|l|}{ Helmintos } \\
\hline Ancilostomídeo & 4 & 2,15 & 2 & 1,58 \\
\hline Ascaris lumbricoides & 17 & 9,13 & 8 & 6,3 \\
\hline Hymenolepis nana & - & - & 2 & 1,58 \\
\hline Strongyloides stercoralis & 1 & 0,53 & - & - \\
\hline Schistosoma mansoni & 1 & 0,53 & - & - \\
\hline Trichuris trichiura & 7 & 3,76 & 1 & 0,79 \\
\hline \multicolumn{5}{|l|}{ Protozoários } \\
\hline Blastocystis spp. & 16 & 8,6 & 13 & 10,31 \\
\hline Chilomastix mesnili & 3 & 1,6 & 3 & 2,38 \\
\hline Endolimax nana & 36 & 19,38 & 16 & 12,69 \\
\hline Entamoeba coli & 19 & 10,2 & 9 & 7,14 \\
\hline Entamoeba histolytica / E. dispar & 6 & 3,22 & 3 & 2,38 \\
\hline Giardia lamblia & 11 & 5,9 & 13 & 10,31 \\
\hline lodamoeba butschilii & 2 & 1,07 & 1 & 0,79 \\
\hline Isospora belli & 1 & 0,53 & - & - \\
\hline Negativo & 63 & 33,4 & 55 & 43,75 \\
\hline Total & 187 & 100 & 126 & 100 \\
\hline
\end{tabular}

Achados semelhantes à da presente pesquisa foram encontrados num estudo de Silva et al. (2016) que analisaram presença de parasitas intestinais em crianças de um Assentamento, maior parte das crianças parasitadas pertenciam a faixa etária entre 5 a 6 anos (33,3\%), seguidas das crianças da faixa etárias entre 7 a 8 e 11 a 12 anos ambas com $23,8 \%$ de positividade. Resultado semelhante também foi encontrado em estudos de Sampaio et al. (2017) na qual a faixa etária mais acometida foi a de pacientes em idade escolar (6 a 15 anos), com $21,7 \%$ das amostras positivas, seguida dos adultos acima de 56 anos, com positividade amostral de $17,8 \%$.

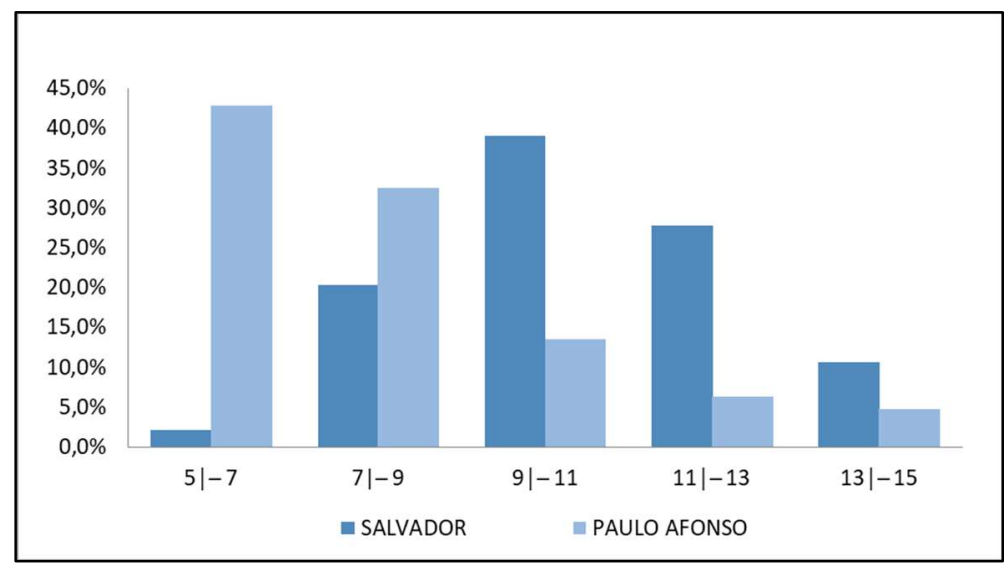

Figura 2: Frequência de enteroparasitoses por faixa etária em crianças oriundas dos municípios de Salvador e Paulo Afonso, BA.

Analisando a frequência de múltiplas infecções de parasitos intestinais nas amostras analisadas, verificou se uma alta frequência de amostras monoparasitadas nos resultados de ambas as cidades, sendo os achados para as respectivas cidades de $31,65 \%$ de amostras monoparasitadas na cidade de Salvador e 31,14\% em Paulo Afonso, já as poliparasitadas encontradas em Salvador com 23,05\% e na cidade de Paulo 
Afonso com 16,98\%. (Figura 3). Resultados semelhantes foram encontrados por estudos desenvolvido por Silva et al. (2016) e Pereira et al. (2018) na qual também identificaram maior prevalência de monoparasitismo nos indivíduos pesquisados.

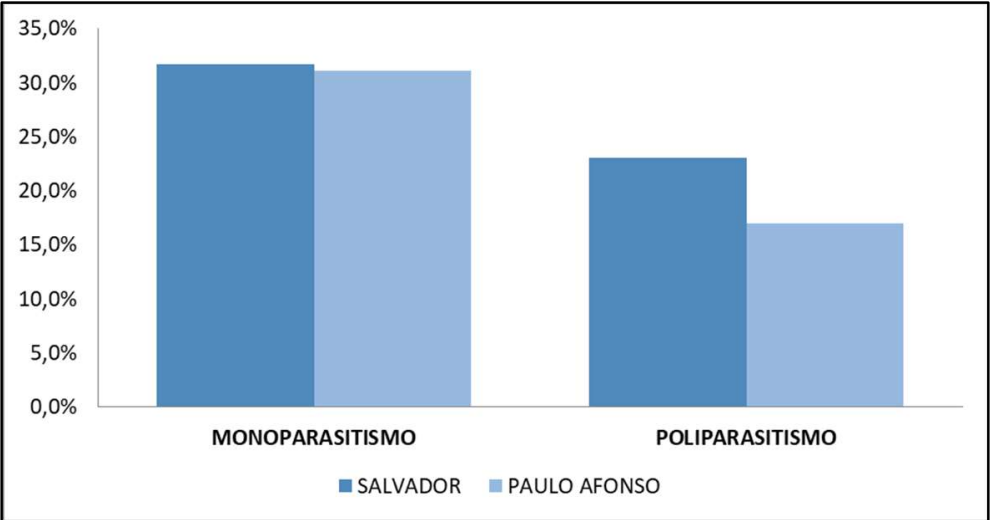

Figura 3: Frequência de múltiplas infecções por parasitos intestinais em crianças dos municípios de Salvador e Paulo Afonso, Bahia.

Com relação de poliparasitismo por faixa etária, observou se uma maior frequência entre a faixa etária de 9-11 anos (39,40\%), 11-13 anos com 24,24\%, 7-9 anos com 21,21\%, 13-15 anos com 12,12\% e 5-7 com 3,03\% da cidade de Salvador, seguido por 5-7 anos (66,66\%), as faixas etárias de 7-9 anos e 9-11 anos ambas com 16,67\% e 11-13 anos e 13-15 anos ambas com 0\% da cidade de Paulo Afonso (Figura 4).

Observa se que em todas as faixas etárias apresentaram indivíduos poliparasitados. Presença de poliparasitismo é um indicador de deficiência de saneamento básico, falta de tratamento de água domiciliar, da aglomeração populacional local e da manutenção de alguns hábitos sanitários tradicionais, como o despejo de dejetos humanos e de resíduos sólidos em áreas ao redor das residências ou próximas a fontes de água de consumo humano (SHAHDOUST et al., 2016).

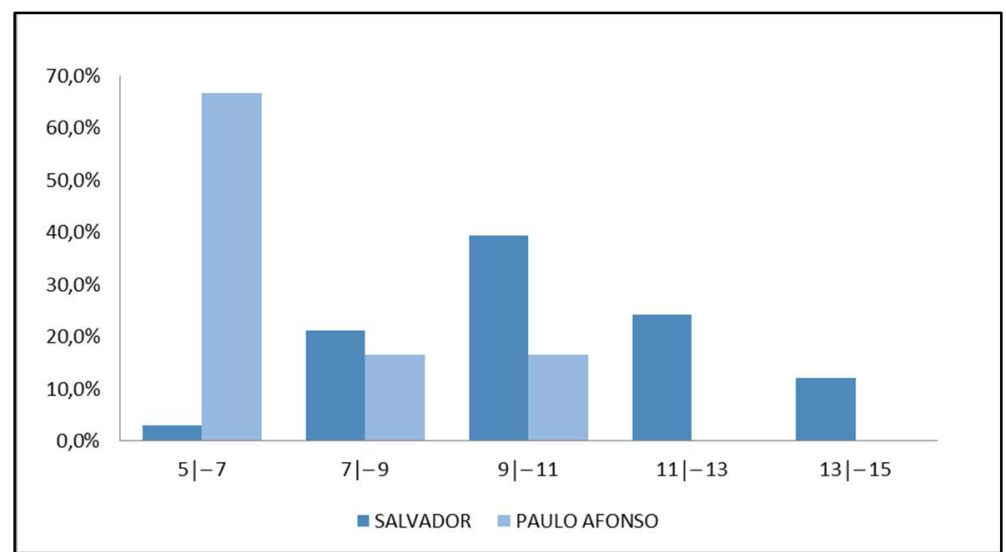

Figura 4: Frequência de poliparasitismo por faixa etária em crianças dos municípios de Salvador e Paulo Afonso, Bahia.

Em relação aos casos de enteroparasitoses por poliparasitismo por sexo, na cidade de Salvador observou a maior frequência no sexo feminino com $53,12 \%$ e masculino com $46,88 \%$. Já na cidade de Paulo Afonso o sexo masculino com $50 \%$ e feminino também com $50 \%$ (Figura 5). A frequência de enteroparasitoses depende do tipo de grau exposição às formas infectantes dos parasitas tais como os ovos, cistos, larvas e trofozoítos. O comportamento das crianças em relação à higienização diária quanto à lavagem das mãos, a 
origem e tratamento da água para consumo, são fatores de exposição que podem ter relação direta com as parasitoses encontradas durante a análise das fezes (ANTUNES et al., 2017).

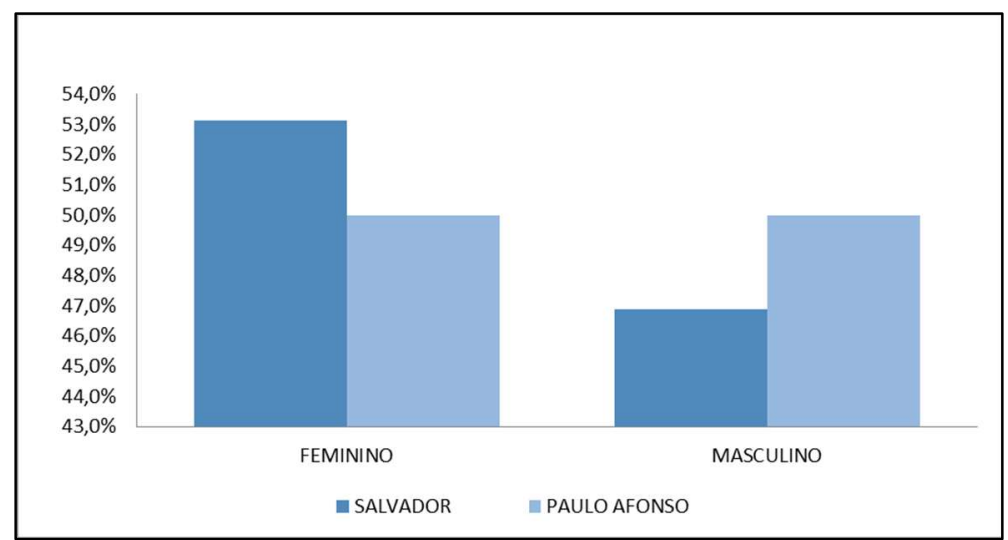

Figura 5: Casos de poliparasitismo por sexo em crianças dos municípios de Salvador e Paulo Afonso, Bahia.

As práticas educativas nas escolas e em casa quando bem aplicadas, levam as pessoas a adquirirem os conhecimento para a prevenção e redução das enteroparasitoses. Sendo de extrema importância para a redução dos índices de enteroparasitas medidas de prevenção, através da melhoria das condições de saneamento básico, socioeconômicas, de educação em saúde e da qualidade nas análises clínicas devem ser asseguradas, como principais medidas de combate a essas doenças (SAMPAIO et al., 2017).

\section{CONCLUSÕES}

Com base nos resultados obtidos observa-se que a prevalência de enteroparasitoses analisadas nas amostras fecais das crianças de 5 a 15 anos das cidades de Salvador e Paulo Afonso na Bahia, foi alta, com predominância no sexo feminino. Este índice pode estar relacionado a fatores como o consumo de água contaminada, a falta de rede de esgoto, coleta de lixo e uma educação sanitária.

Ressalta se que se deve ver com cautela, uma vez que o método de diagnóstico empregado não é o mais adequado para detectar esses parasitas, sugerindo que possivelmente têm prevalências maiores que as aqui relatadas. Embora o parasitismo intestinal ainda represente um grave problema de saúde pública no Brasil, ao longo do tempo esse problema passou a fazer parte do grupo de doenças negligenciadas, por motivos de falta de informação, e por ser considerada por muitos como 'doenças de gente pobre'.

A educação em saúde para crianças é um fator essencial para a prevenção e controle de enteroparasitoses, especialmente considerando as características da doença durante a infância que pode se definir como: em alta prevalência, alta porcentagem de resistência ao tratamento clinico, altas taxas de eliminação de ovos, altos níveis de reinfecção bem como os hábitos e práticas de higiene das mesmas. Os dados sugerem a realização de pesquisas mais aprofundadas e exames de fezes de rotina com faixa etária que corresponde as crianças de 5 a 13 anos nas cidades pesquisadas, pelo alto índice de infecções diagnosticado, bem como indica possíveis problemas socioeconômicos na região estudada. 


\section{REFERÊNCIAS}

AULER, M. E.; CAMPOS, L. A.; HORST, J. A. E.; SANTOS, T. B. dos; MIYAHARA, C. T. S.; PAULA, C. R.; RUIZ, L. S.; GRANDA, R. F.; FIGUEIREDO, D. L. A.. Saúde itinerante nos centros municipais de educação infantil do município de Guarapuava/PR; os desafios da promoção da saúde em crianças expostas a doenças parasitárias. Arq. Cienc. Saúde UNIPAR, Umuarama, v.22, n.1, p.33-41, 2018.

ANDRADE, A. O.; SÁ, A. R. N.; BEZAGIO, R. C.. Prevalência de parasitoses intestinais em crianças de um centro municipal de educação infantil de Campo Mourão, PR/Brasil. Revista UNINGÁ Review, v.29, n.3, p.36-41, 2017.

ANTUNES, A. S.; LIBARDONI, K. S. B.. Prevalência de enteroparasitoses em crianças de creches do município de Santo Ângelo, RS. Revista Contexto \& Saúde, v.17, n.32, 2017.

FIOCRUZ. Doenças do aparelho digestivo: Parasitoses intestinais. Universidade Aberta do SUS, 2019.

LACERDA, J. S.; JARDIM, C. M. L.. Estudo da prevalência de parasitoses intestinais em pacientes de um laboratório privado de Araçatuba/SP. Revista Saúde UniToledo, Araçatuba, v.1, n.1, p. 107-120, 2017.

NAVONE, G. T.; ZONTA, M. L.; COCIANCIC, P.; GARRAZA, M. GAMBOA, M. I.; GIAMBELLUCA, L. A.; DAHINTEN, S.; OYHENART, E. E.. Estudio transversal de las parasitosis intestinales en poblaciones infantiles de Argentina. Rev. Panam Salud Publica, v.41, n.e24, 2017.
PEREIRA, G. L. T.; RIBEIRO, C. A.; COSTA, I. O.; SILVA, J. N. C.; CALADO, L. S. O.; NUNES, B. R. M.; AGUIAR, J. J. S.; RODRIGUES, F. F. G.; MOTA, M. L.. Prevalência de infecções parasitárias intestinais oriundas de crianças residentes em áreas periféricas, município de Juazeiro do Norte - Ceará. Rev. Interfaces, v.5, n.14, p.21-27, 2017.

SANTOS, P. H. S.; BARROS, R. C. S.; GOMES, K. V. G.; NERY, A. A.; CASOTTI, C. A.. Prevalência de parasitoses intestinais e fatores associados em idosos. Revista Bras. Geriatr. Gerontol., v.20, n.2, p.244-253, 2017. DOI: https://doi.org/10.1590/1981-22562017020.160137

SAMPAIO, J. P. S.; BARROS, V. C.. Prevalência de enteroparasitoses em pacientes atendidos em uma unidade pública de saúde no município de Beneditinos/PI. Jornal Interdisciplinar de Biociências, v.2, n.1, 2017.

SILVA, E. P.; CARVALHO, W. R. C.; FIRMO, W. C. A.. Estudo comparativo da ocorrência de parasitoses intestinais em crianças de duas comunidades da zona rural de Paulo Ramos/MA, Brasil. Revista UNINGÁ Review, v.27, n.2, p.1521, 2016.

SBMFC. Sociedade Brasileira de Medicina de Família e Comunidade. Parasitoses intestinais. Rio de Janeiro: SBMFC, 2019.

SHAHDOUST, S.; NIUUATI, M.; HAGHIGHI, A.; AZARGASB, E.; KHATAMINEJAD, M. R.. Prevalence of intestinal parasites in referred individuals to the medical centers of Tonekabon city, Mazandaran province. Gastroenterology and Hepatology from Bed to Bench, v.9, n.1, p.S75-S79, 2016.

A CBPC - Companhia Brasileira de Produção Científica (CNPJ: 11.221.422/0001-03) detém os direitos materiais desta publicação. Os direitos referem-se à publicação do trabalho em qualquer parte do mundo, incluindo os direitos às renovaç̃os, expansões e disseminacões da contribuicão, bem como outros direitos subsidiários. Todos os trabalhos publicados eletronicamente poderão posteriormente ser publicados em coletâneas impressas sob coordenação da Sustenere Publishing, da Companhia Brasileira de Produção Científica e seus parceiros autorizados. Os (as) autores (as) preservam os direitos autorais, mas não têm permissão para a publicação da contribuição em outro meio, impresso ou digital, em português ou em tradução. 